\title{
Perioperative pulmonary aspiration and regurgitation without aspiration in adults: a retrospective observational study of 166,491 anesthesia records
}

\author{
Jiacheng Sun", Guohua Wei", Liuqing Hu", Cunming Liu, Zhengnian Ding \\ Department of Anesthesiology and Perioperative Medicine, First Affiliated Hospital with Nanjing Medical University, Nanjing, China \\ Contributions: (I) Conception and design: Z Ding; (II) Administrative support: C Liu; (III) Provision of study materials or patients: J Sun, G Wei, \\ L Hu; (IV) Collection and assembly of data: J Sun, G Wei, L Hu; (V) Data analysis and interpretation: Z Ding, J Sun; (VI) Manuscript writing: All \\ authors; (VII) Final approval of manuscript: All authors. \\ "These authors contributed equally to this work. \\ Correspondence to: Dr. Zhengnian Ding. Department of Anesthesiology and Perioperative Medicine, First Affiliated Hospital with Nanjing Medical \\ University, Nanjing 210029, China. Email: zhengnianding@njmu.edu.cn.
}

Background Pulmonary aspiration (PA) of gastric contents is a rare but serious perioperative complication. Recent studies focused on pediatric patients, but over a decade has passed since the latest incidence and outcome in adult population have been reported. Patients who experienced regurgitation without aspiration were rarely mentioned. Besides, our department proposed a modified rapid sequence induction (RSI) protocol in 2018 and its preventive effect remained to be examined.

Methods: A total of 166,491 anesthesia records from March 2015-October 2020 were reviewed. Outcomes from regurgitation events were classified as PA or regurgitation without aspiration following strict criteria. Available information including demographics, anesthetic managements, surgical procedures, and other medical records were reviewed for analysis.

Results: Among the 166,491 anesthesia records, 20 patients had PA (1:8,325), and 20 had regurgitation without aspiration $(1: 8,325)$. The morbidity of PA was $1: 16,649$, and the mortality was $1: 55,497$. During anesthesia induction, $76.0 \%$ of regurgitation events developed aspiration, and the remaining $24.0 \%$ had regurgitation without aspiration. But prior to anesthesia induction, only $10.0 \%$ regurgitation events developed aspiration. Emergency procedures were associated with serious risks of PA (OR: 27.1, 95\% CI: 10.8-68.0) and regurgitation without aspiration (OR: 83.0, 95\% CI: 24.3-283.1) compared with elective procedures. The highest incidence of pulmonary aspiration was observed in bronchoscopy procedures $(2 / 1,747)$. The modified RSI reduced the incidence of regurgitation events during induction in emergency procedures but did not show significant advantages over classic protocol (0:1,055 versus 12:4,469, $\mathrm{P}=0.139)$ possibly due to insufficient sample size. The sample size required for future study was estimated based on the current data.

Conclusions: The incidence of pulmonary aspiration and regurgitation without aspiration was low, especially in elective cases. Regurgitation during anesthesia induction had mostly developed aspiration. Further evaluation of the effect of modified RSI protocol needs a large sample size.

Keywords: Pulmonary aspiration; regurgitation; anesthesia; rapid sequence induction (RSI)

Submitted Dec 01, 2020. Accepted for publication Feb 03, 2021.

doi: 10.21037/apm-20-2382

View this article at: http://dx.doi.org/10.21037/apm-20-2382 


\section{Introduction}

Pulmonary aspiration (PA) of gastric contents is a rare but dangerous perioperative complication that potentially causes anesthesia-related morbidity and mortality $(1,2)$. PA can result in aspiration pneumonia, aspiration pneumonitis, acute respiratory distress syndrome (ARDS), and subsequent bacterial infection through particle-, acid- and bacteriarelated mechanisms (2). Over the recent years, studies on PA have concentrated on pediatric patients (3-6). To our knowledge, no large retrospective observational study on PA incidence in adults or all ages has been published since 2009 (7,8). Considering the increasing awareness of PA prevention and the development of treatment, recent and detailed data of PA are valuable.

Although gastric contents regurgitation is the premise of PA, regurgitation cases without aspiration are rarely mentioned possibly due to the low risk of complications. In this work, the incidence of $\mathrm{PA}$ and regurgitation without aspiration was investigated to determine their proportions in different perioperative phases. The findings may help estimate the probability of aspiration and choose suitable treatments post-regurgitation, which is especially helpful when aspiration cannot be confirmed immediately.

The rapid sequence induction (RSI) protocol used for prevention of $\mathrm{PA}$ is quite similar worldwide, but the details vary among institutions and countries $(9,10)$. Conducting a prospective randomized study to support prevention strategies is difficult due to well-described ethical, methodological, and practical limitations. Hence, no international consensus has been reached about the anesthesia induction protocol in patients at risk of PA. In our department, the RSI protocol was modified and adopted by the newly authorized junior attending doctors from March 1, 2018. The effect of such modification was evaluated.

A total of 166,491 cases were reviewed in a 5.5-year period. The primary objective was to investigate the incidence and outcome of $\mathrm{PA}$ and regurgitation without aspiration in our institution. The secondary objective was to evaluate the effect of modified RSI protocol in preventing regurgitation events during anesthesia induction in emergency cases. Risk factors, anesthetic management, and treatment were also discussed. We present the following article in accordance with the STROBE reporting checklist (available at http://dx.doi.org/10.21037/apm-20-2382).

\section{Methods}

\section{General materials}

This retrospective observational study was approved by the Human Research Ethics Committee of the First Affiliated Hospital with Nanjing Medical University (2020SR-520), and informed consent was waived. The study was performed in compliance with the ethical principles outlined in the Declaration of Helsinki (as revised in 2013). Anesthesia records during the 5.5-year study period (March 2015-October 2020) were exported from the Electronic Anesthesia Record System of our institution. If data on age, department, or diagnosis were absent, the admission notes were reviewed to recover the missing information. The absence of patient ID, American Society of Anesthesiologist (ASA) grade, or the record of intraoperative events were unrecoverable. These anesthesia records were classified into invalid records and thus excluded. According to inconsistent regulations and quality control criteria, the procedures in branch hospital (mainly comprised of gynecological, obstetric, and breast procedures) were also excluded.

\section{Study methods}

Two separate databases were used to identify the occurrence of gastric contents regurgitation and PA. First, the following keywords were retrieved in the exported data of Electronic Anesthesia Record System for relevant records: "aspiration," "regurgitation," "vomit," "vomitus," "gastric contents," and "gastric fluid." These cases were then searched in the Hospital Information System. Medical data on admission, hospitalization course, operation, discharge, and perioperative imaging data were reviewed. The records containing keywords but unrelated to regurgitation were excluded, including explicitly documented postoperative nausea and vomiting, respiratory secretions, and gastric tube suctioning for surgical demands. Two researchers separately identified each case and made the diagnosis. Cases with conflicts were further reviewed and discussed to reach an agreement.

As prescribed by the department, suctioning from oral and artificial airway (when established) was implemented when gastric contents were observed in oropharynx or laryngopharynx. Bronchoscopy was optional. Regurgitation was identified as perioperative when it occurred in the 
period from the patient entering the operating room to 2 hours after the completion of procedure $(4,11)$. The perioperative period was further divided into four phases (prior to induction, induction, maintenance, and emergence). For cases with documented regurgitation, the existence of PA was evaluated following the diagnostic criteria below.

\section{Definite aspiration}

Regurgitation was documented. Evidence of any nonrespiratory secretions (bilious or particulate) in the lower respiratory tract was detected by suctioning or bronchoscopy. When non-respiratory secretion was not found or the airway was not examined, this diagnosis could also be supported by a newly reported infiltrate in chest radiograph within 24 hours post-operation, provided that other causes were excluded $(11,12)$.

\section{Suspected aspiration}

Regurgitation was documented. The patient developed intraoperative episodes of hypoxia (pulse oxygen saturation $<90 \%$ ), but no positive result was found by suctioning, bronchoscopy, or chest radiograph $(11,13)$.

\section{Regurgitation without aspiration}

Regurgitation was documented. The patient data did not meet the criteria for definite and suspected aspiration.

Patient's age, gender, ASA grade, type of admission, surgical department, diagnosis, and anesthetic management were obtained from the Electronic Anesthesia Record System. Postoperative course, image data, and outcome were obtained from the Hospital Information System. The risk factors of $\mathrm{PA}$ and regurgitation were retrieved from the anesthesia records and admission notes to avoid omission. In morbidity calculation, patients who required intensive care unit (ICU) admission and respiratory support, or developed pulmonary complications because of PA were included (11). Comparison between modified and classic RSI protocol was conducted among emergency cases (details of protocols are shown in Table S1).

\section{Statistical analysis}

All the anesthesia records of procedures performed in the headquarters of the institution during study period were included, except for the invalid records and pediatrics (14). Data were analyzed using IBM SPSS software, version 24.0 (IBM Corp., Armonk, NY, USA). Continuous variable with non-normal distribution (age) and ranked data (ASA grade and number of risk factors) were compared by MannWhitney U test. Categorical variables (gender, type of admission, type of anesthesia, and time of occurrence) were analyzed by Chi-square test or Fisher's exact test as appropriate. Odds ratio (OR) and $95 \%$ confidence interval (CI) were calculated to evaluate the effect of gender, type of admission, and shift (day or night) on the occurrence of $\mathrm{PA}$ and regurgitation without aspiration. $\mathrm{P}$ value $<0.05$ was considered as significant. Bonferroni correction was used for pairwise comparison.

\section{Results}

A total of 236,387 anesthesia records from March 2015 to October 2020 were exported from the Electronic Anesthesia Record System. After the exclusion of invalid records $(8,424$ cases), procedures in the branch hospital (54,522 cases), and pediatrics (6,950 cases), 166,491 cases remained for further analysis. Demographic data of the study population are presented in Table S2. The proportion of male to female was $92,300 / 74,191$ (1.24:1), and emergency procedures constituted $6.4 \%$ of the total population.

\section{Incidence of $\mathrm{PA}$ and regurgitation without aspiration}

The keywords "aspiration", "regurgitation", "vomit”, "vomitus", "gastric contents", or "gastric fluid" were found in 300 records. After cautious evaluation, 40 patients were identified experiencing regurgitation events and classified into definite aspiration, suspected aspiration, and regurgitation without aspiration (Figure 1). Comparison of demographic data is shown in Table 1. The remaining 260 cases containing keywords correlating to $\mathrm{PA}$ or regurgitation were excluded (see Table S3 for exclusion reasons). During anesthesia induction, $76.0 \%$ of regurgitation events developed aspiration. However, prior to anesthesia induction, only $10 \%$ regurgitation events developed aspiration (Table 2). Details of the 40 patients are presented in Table S4.

\section{Surgical specialty}

The surgical specialized incidences are shown in Table S5. The highest incidence of PA was observed in bronchoscopy procedures $(2 / 1,747)$. The two patients received tracheal stent placement to relieve tracheal stenosis. One patient had a full stomach, and the other had a history of 


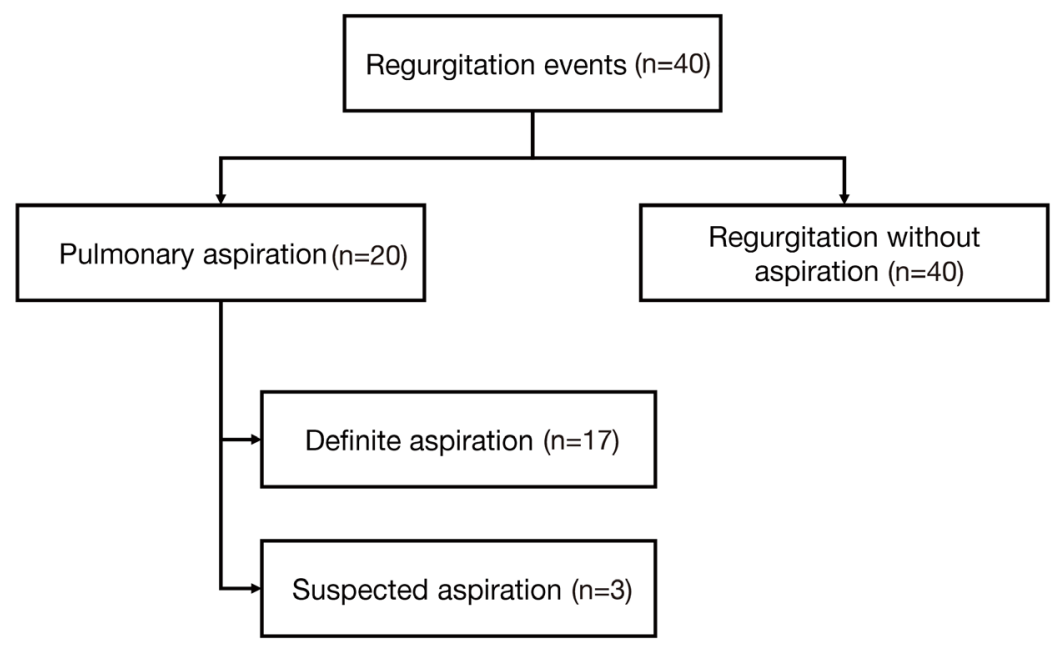

Figure 1 Patients with regurgitation events. Regurgitation events were classified into pulmonary aspiration and regurgitation without aspiration. Cases with pulmonary aspiration included those with definite aspiration and suspected aspiration.

esophageal surgery. A total of 13 patients with PA and 12 with regurgitation without aspiration were scheduled for abdominal surgery.

\section{Risk factors}

Male patients and emergency procedure were associated with high risks of PA and regurgitation without aspiration (Table 3). For emergency procedures, nightshift cases had a higher risk of PA compared to dayshift, and the respective result of regurgitation without aspiration was not significantly different (Table 4). High ASA grades were found in patients with PA and regurgitation without aspiration ( $\mathrm{P}<0.001$ for both) (Table $\mathrm{S} 6)$.

Risk factors of perioperative gastric contents regurgitation and PA were found in 36 out of the 40 patients (30 emergency procedure, 20 recent meal, 8 acute abdomen, 7 depressed level of consciousness, 5 intestinal obstruction, 4 body mass index $>27,3$ previous esophageal surgery, 3 sepsis, 2 incarcerated inguinal hernia, and 1 renal failure). A significantly lower number of risk factors were found in patients with $\mathrm{PA}$ compared to those with regurgitation without aspiration ( $\mathrm{P}=0.020$, Table 1$)$.

Prior to anesthesia induction, regurgitation of two cases occurred during nasogastric tube insertion. During maintenance, regurgitation was observed upon transfer to Trendelenburg position in one patient, during gastrointestinal manipulation in two patients and occurred 4 minutes after intubation in an emergency patient with a full stomach. Regurgitation during emergence of anesthesia was documented in one patient with cerebral hemorrhage who was scheduled to return to the ward with endotracheal tube (ETT). None of these patients developed aspiration.

\section{Classic and modified RSI protocol}

A total of 4,469 emergency patients received classic RSI, and 12 of them developed regurgitation events during anesthesia induction. None of the 1,055 patients receiving modified RSI experienced regurgitation during induction ( $\mathrm{P}=0.139$ by Fisher's exact test). On the basis of the current data, a sample size composed of 7,484 cases for modified RSI protocol and 14,968 cases for classic RSI protocol was calculated to detect statistical difference (Table S7).

\section{Treatment}

Among the 10 patients who experienced PA or regurgitation without aspiration prior to induction, 2 received fiberoptic bronchoscopy after intubation, and the rest were administered with blind suctioning. Hydrocortisone was used in 4 patients.

Regurgitation events were documented in 25 cases during anesthesia induction. Sixteen of 19 patients with PA and one of 6 patients with regurgitation without aspiration received additional one or more treatments apart from blind suctioning in the operating room. Hydrocortisone was the most frequently used and was applied in 14 patients. 
Table 1 Demographic data of patients diagnosed with perioperative pulmonary aspiration and regurgitation without aspiration

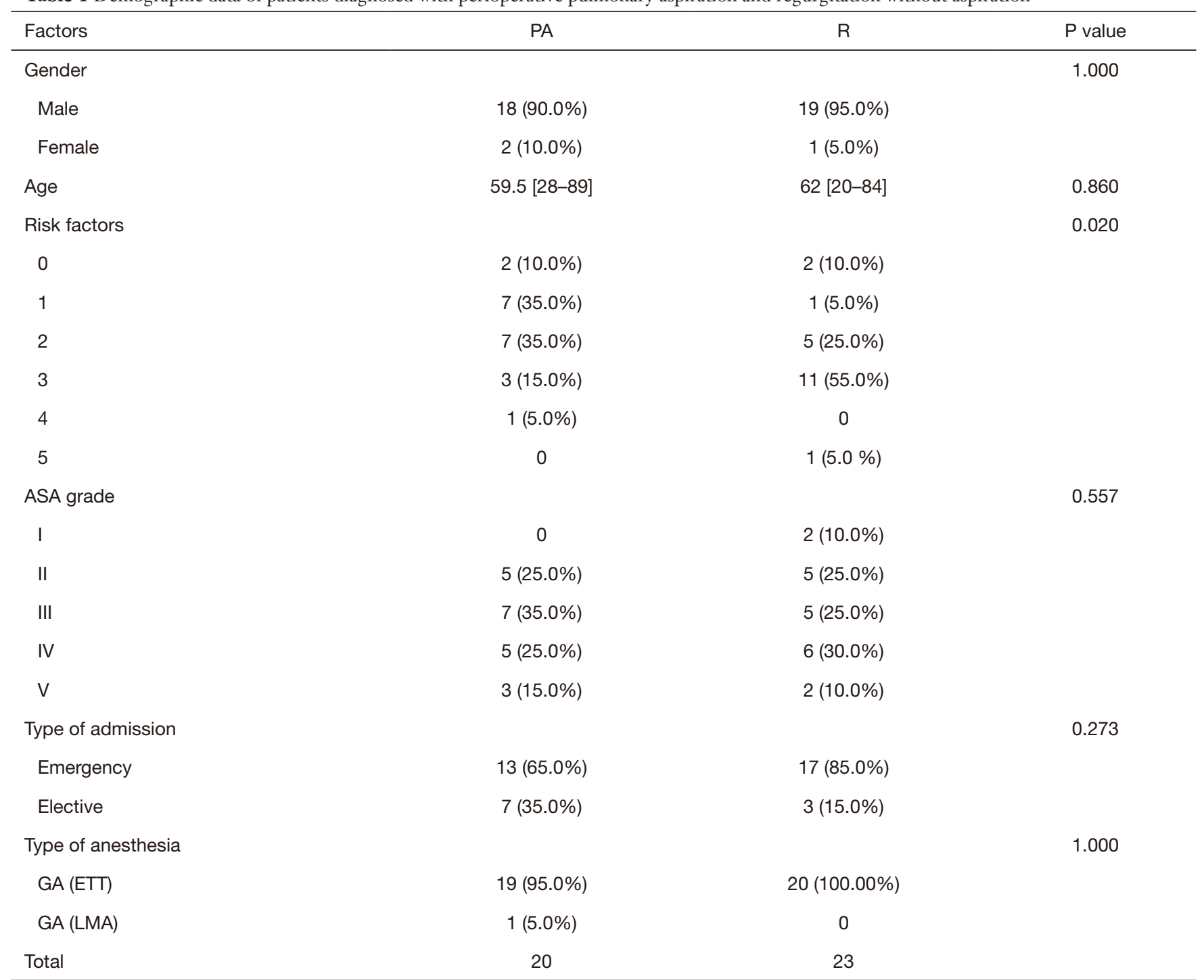

Age is presented as median [range]. PA, pulmonary aspiration; R, regurgitation (without aspiration); GA, general anesthesia; ETT, endotracheal tube; LMA, laryngeal mask airway.

Table 2 Proportions of pulmonary aspiration and regurgitation without aspiration in different perioperative phases

\begin{tabular}{lcccc}
\hline Outcomes & Prior to induction & Induction & Maintenance & Emergence \\
\hline PA & $1(10.0 \%)$ & $19(76.0 \%)$ & 0 & 0 \\
R & $9(90.0 \%)$ & $6(24.0 \%)$ & $4(100 \%)$ & $1(100 \%)$ \\
Total & 10 & 25 & 4 & 1 \\
\hline
\end{tabular}

Pairwise comparison showed significant difference between "prior to induction" and "induction", Bonferroni-corrected P=0.006. PA, pulmonary aspiration; $\mathrm{R}$, regurgitation (without aspiration). 
Table 3 The effect of gender and type of admission on the occurrence of pulmonary aspiration and regurgitation without aspiration

\begin{tabular}{|c|c|c|c|c|}
\hline Factors & PA/non-PA (n) & OR $(95 \% \mathrm{Cl})$ & R/non-R (n) & OR $(95 \% \mathrm{Cl})$ \\
\hline Male & $18 / 92,282$ & $7.2(1.7-31.2)$ & $19 / 92,281$ & $15.3(2.0-114.1)$ \\
\hline Female & $2 / 74,189$ & & $1 / 74,190$ & \\
\hline \multicolumn{5}{|c|}{ Type of admission } \\
\hline Elective & $7 / 155,823$ & & $3 / 155,827$ & \\
\hline
\end{tabular}

PA, pulmonary aspiration; $\mathrm{R}$, regurgitation (without aspiration); OR, odds ratio; $\mathrm{Cl}$, confidence interval.

Table 4 Risks of pulmonary aspiration and regurgitation without aspiration between nightshift and dayshift emergency procedures

\begin{tabular}{lcccc}
\hline Shifts & PA/non-PA $(\mathrm{n})$ & OR $(95 \% \mathrm{Cl})$ & $\mathrm{R} / \mathrm{non}-\mathrm{R}(\mathrm{n})$ & $\mathrm{OR}(95 \% \mathrm{Cl})$ \\
\hline Nightshift & $10 / 4,637$ & $4.3(1.2-15.7)$ & $11 / 4,636$ & $2.4(0.9-6.4)$ \\
Dayshift & $3 / 6,011$ & & $6 / 6,008$ & \\
\hline
\end{tabular}

$\mathrm{PA}$, pulmonary aspiration; $\mathrm{R}$, regurgitation (without aspiration); OR, odds ratio; $\mathrm{Cl}$, confidence interval.

Eight individuals received suctioning under bronchoscopy, including two cases of bronchoscopy procedure in which the surgeon cleaned the airway.

For the 5 patients with regurgitation during maintenance or emergence, gastric contents in oral and pharynx cavity were suctioned when regurgitation was detected. No evidence of aspiration was found through either suctioning from ETT or postoperative information.

\section{Outcomes}

Seven of the 20 patients with PA were extubated within 4-144 minutes with an average of 59.0 minutes following the end of procedure. Five patients were transferred to ICU with mechanical ventilation support due to $\mathrm{PA}$, and eight patients returned with ETT as scheduled. Ten patients required ICU admission, respiratory support, or developed pulmonary complications because of PA. Among them, six patients developed respiratory failure following surgery, and three recovered. The death of three patients was attributed to aspiration (details are shown in Table S8).

The outcomes of the 20 patients with regurgitation without aspiration were also reviewed. Among them, 11 were extubated within 5-53 minutes with an average of 23.3 minutes following the end of procedure. The remaining nine patients returned with ETT as planned. Three deaths were attributed to primary diseases.

\section{Discussion}

This retrospective study identified 20 patients with perioperative PA in 166,491 anesthesia records, thus resulting in an incidence of $1: 8,325$. Ten patients required intensive care, respiratory support, or developed pulmonary complications, and the morbidity was $1: 16,649$. Three deaths were mainly attributed to PA, and the mortality was 1:55,497. Regurgitation without aspiration was found in 20 patients $(1: 8,325)$, none of whom developed pulmonary complications.

Two large studies have described the incidence and outcome of perioperative PA in adult population. Warner et al. reported an incidence of 1:3,216, morbidity of $1: 11,971$, and mortality of 1:71,829 during 1985-1991 (11). Sakai et al. reported an incidence of 1:7,103, which was approximately half that of the former but the morbidity and mortality were similar (14). The PA incidence in emergency procedures was different between the former $(1: 895)$ and the latter $(1: 4,474)$ studies. A possible reason proposed by the latter study is the regular allocation of senior handson providers during off-hour shifts, when most emergency procedures are conducted (14).

This work followed the strict diagnostic criteria of PA and the definition of morbidity proposed by Warner et al. (11). As is shown in Table 5, the incidence and morbidity in the present research are the lowest among the three studies, whereas the mortality is slightly higher. 
Table 5 Comparison among the studies on pulmonary aspiration focusing on adult populations

\begin{tabular}{lccccccc}
\hline Study period & $\begin{array}{c}\text { Number of } \\
\text { patients }\end{array}$ & Incidence & Morbidity & Mortality & $\begin{array}{c}\text { Incidence } \\
\text { (emergency) }\end{array}$ & $\begin{array}{c}\text { Incidence } \\
\text { (elective) }\end{array}$ & Reference \\
\hline $1985-1991$ & 215,488 & $1: 3,216$ & $1: 11,971$ & $1: 71,829$ & $1: 895$ & $1: 3,886$ & Warner et al. (11) \\
$2001-2004$ & 99,441 & $1: 7,103$ & $1: 16,573$ & $1: 99,441$ & $1: 4,474$ & $1: 7,541$ & Sakai et al. (14) \\
2015-2020 & 166,491 & $1: 8,325$ & $1: 16,649$ & $1: 55,497$ & $1: 820$ & $1: 22,261$ & Present study \\
\hline
\end{tabular}

The current PA incidence in emergency procedures is consistent with that of Warner et al. but higher than that of Sakai et al. In addition, the PA incidence in elective cases is quite low. In our institution, senior attending doctors are also routinely arranged to take charge during off-hour shifts. These differences could be attributed to several reasons. The proportion of patients $\geq 65$ years in our study was $28.5 \%$, which was lower than that in previous studies (approximately $40 \%$ ), and the constituent ratios of surgical departments vary among these reports. Over the past decades, preoperative fasting and evaluation for elective patients have become increasingly cautious; however, major improvements are difficult to achieve in emergency cases. Besides, the proportions of patients under PA risk may be different among these studies.

The incidence of regurgitation without aspiration has been ignored by most researchers. Beck et al. reported the regurgitation (without aspiration) incidence of 1:390 in pediatric patients, which is approximately twice that of PA (5). In this study, the incidence of PA and regurgitation without aspiration happened to be equal $(1: 8,325)$. Owing to omitted reporting, the incidence of regurgitation without aspiration may be underestimated in such retrospective studies. In the 40 patients, the regurgitation events during anesthesia induction caused a high percentage of aspiration. Most regurgitation events prior to induction were confirmed with regurgitation without aspiration. This finding is in accordance with the fact that anesthetics reduce the esophageal sphincter pressure and impair the protective airway reflexes, while awake patients maintain the ability to avoid aspiration (15). The difference was expected, but the lack of evidence from a large population had limited the results. In clinical practice, the diagnosis of PA depends on airway examination or complications. This sometimes results in hysteresis. Thus, the choice of immediate treatment may be blind. The above outcomes indicate that regurgitation during anesthesia induction could possibly develop aspiration, whereas most regurgitation events prior to anesthesia induction are safe. This finding helps in evaluating the possibility of aspiration and choosing treatment when aspiration cannot be immediately diagnosed.

Whether any motivator promotes PA under the premise of regurgitation remains unclear. A study performed on a life-like manikin suggested that a large regurgitant gastric fluid volume and sniffing position may contribute to PA occurrence (16). However, conducting relevant experimental studies on humans is impossible. In the present retrospective study, the gender, age, and ASA grade were comparable between the patients with PA and regurgitation without aspiration. More data are needed to evaluate whether there is a difference between patients with $\mathrm{PA}$ and regurgitation without aspiration in the number of risk factors.

Multiple risk factors promote the occurrence of gastric contents regurgitation and PA $(2,12)$. The incidence of PA in emergency cases was 1.7-4.3 times higher than that of elective cases $(11,14)$ in adults and 4.3-12.1 times in pediatrics $(3,17)$. However, our study found a great difference of 27.1-fold. The low incidence in elective patients of our institution may contribute to this difference. Olsson et al. reported that the PA incidence was 2.9-fold higher in male patients than in females (18). We also found a 7.2-fold difference in this work. A high PA incidence of nightshift was also reported in emergency cases, which is also in accordance with a previous report (18).

ASA grade and diabetes are associated with perioperative regurgitation events but are usually not considered as risk factors. Patients with PA had high ASA grades (11); however, this condition is influenced by complex factors. An acknowledged standard to associate ASA grade with PA risk remains lacking. Patients with diabetes, especially diabetes-related autonomic neuropathy, retinopathy, and nephropathy, are at increased risk of delayed stomach emptying (19,20). Zhou et al. recommended preoperative ultrasound assessment of gastric content for patients with diabetes duration $\geq 6$ years, prokinetic drugs, and RSI (21).

The anatomical structure and mechanism of PA 
have been summarized $(15,22)$. Several methods were recommended for prevention, including preoperative fasting, antiacids, prokinetics, gastric drainage through nasogastric tube, awake tracheal intubation, and RSI (15). Nasogastric tube is widely used to control gastric volume before anesthesia. However, this technique needs cautious application because it can reduce lower and upper esophageal sphincter pressure (23). In our study, the insertion of nasogastric tube induced regurgitation in two cases. These methods are mainly based on indirect outcomes without sufficient evidence of incidence, morbidity, and mortality of PA in large population. The obstructions include limited application, extremely low incidence, presence of confounding factors, and limitation of ethical principles.

Although different forms of modified or controlled RSI protocols have been proposed, reaching a breakthrough in RSI optimization is difficult due to the above obstructions $(24,25)$. In our study, the modified RSI protocol reduced the incidence of regurgitation events during anesthesia induction (0:1,055 versus 12:4,469), but the difference was not significant. On the basis of this outcome, a huge sample size was calculated to detect statistical difference. The potential advantage of the modified RSI protocol will be the focus of our future work.

Different treatments for PA have been outlined (2). The methods incorporated in our findings, including suctioning (blind or under bronchoscopy), bronchial lavage, PEEP, and bronchodilators, have been proven beneficial. Bronchoscopy should be performed frequently when PA is suspected. However, corticosteroids, which have no benefit $(26,27)$, were applied intraoperatively in 18 of the 40 patients when aspiration was confirmed or suspected. A chest radiograph assessment after aspiration, even if suspected, is recommended (28). Among our patients with PA or regurgitation without aspiration, only $30.0 \%(12 / 40)$ received chest radiograph within 3 days post-operation.

$\mathrm{PA}$ in patients receiving bronchoscopy procedures is rarely discussed (18). In our study, two patients scheduled for bronchoscopy had experienced PA. Both of them had dyspnea and were diagnosed with tracheal stenosis. Lack of respiratory coordination might have contributed to this condition (14). Furthermore, most bronchoscopy procedures are conducted through laryngeal mask airway, let alone rigid tracheoscopy. That means ETT is sometimes impracticable for emergency cases or high-risk patients in this kind of procedures, thus airway protection is lacking. Hence, the evaluation of PA risk is highly necessary for bronchoscopy patients.

Regurgitation may occur after intubation, but PA rarely happens under the protection of ETT. We recommend to pay attention to the ETTs that were established outside the operating room. Patient No. 23 was transferred to operating room under mechanical ventilation, and his gastric contents overflowed from the mouth and nose. Prior to induction, a shallow ETT depth was found. This condition indicated that the airtightness of the balloon might have been lost, or the tube has escaped the trachea. The patient was at risk of aspiration with a dislocated ETT. After the adjustment of ETT depth under laryngoscopy, bronchoscopy results revealed no abnormality. This case alerts us that the patient under the intubation status is not completely free from the risk of aspiration. Examining the condition of the ETT that inserted outside the operating room remains necessary.

According to past experience, the patients without sustained pulmonary complications in a 2-hour period after extubation can be transferred to the normal ward (29). However, we recorded a delayed occurrence of hypoxemia for over 2 hours after extubation in patient No. 19, who finally died of PA (Table S8). We recommend to pay attention to early asymptomatic patients, especially those already confirmed with aspiration, because of their possible risk of severe complications.

This study has several limitations. The regurgitation events were identified by searching specific keywords in the Electronic Anesthesia Record System. Regurgitation events prior to and during anesthesia induction are seriously considered and well documented in most cases. However, minor regurgitation events, such as regurgitation after ETT established, are considered safe and sometimes unrecorded. Undetected regurgitation and aspiration also exist. Therefore, this study may underestimate the incidence of regurgitation events. In the anesthesia records, the detailed information of fasting was not available because only "fasted" or "not fasted" was documented after evaluation. Among the 40 patients, 17 were scheduled to be transferred to ICU or return with ETT. As a result, our study may underestimate the morbidity. The present data are not suitable for logistic regression analysis because multiple predictors remain to be considered while the number of patients with regurgitation events is insufficient for the regression.

\section{Conclusions}

Perioperative PA is a rare complication. Its morbidity and mortality in our institution were comparable to the reports 
of other centers. Regurgitation during anesthesia induction could mostly develop aspiration, whereas regurgitation prior to induction seldom causes aspiration. Further evaluation of the effect of modified RSI protocol needs a large sample size.

\section{Acknowledgments}

Funding: None.

\section{Footnote}

Reporting Checklist: The authors have completed the STROBE reporting checklist. Available at http://dx.doi. org/10.21037/apm-20-2382

Data Sharing Statement: Available at http://dx.doi. org/10.21037/apm-20-2382

Peer Review File: Available at http://dx.doi.org/10.21037/ apm-20-2382

Conflicts of Interest: All authors have completed the ICMJE uniform disclosure form (available at http://dx.doi. org/10.21037/apm-20-2382). The authors have no conflicts of interest to declare.

Ethical Statement: The authors are accountable for all aspects of the work in ensuring that questions related to the accuracy or integrity of any part of the work are appropriately investigated and resolved. This retrospective observational study was approved by the Human Research Ethics Committee of the First Affiliated Hospital with Nanjing Medical University (2020-SR-520), and informed consent was waived. The study was performed in compliance with the ethical principles outlined in the Declaration of Helsinki (as revised in 2013).

Open Access Statement: This is an Open Access article distributed in accordance with the Creative Commons Attribution-NonCommercial-NoDerivs 4.0 International License (CC BY-NC-ND 4.0), which permits the noncommercial replication and distribution of the article with the strict proviso that no changes or edits are made and the original work is properly cited (including links to both the formal publication through the relevant DOI and the license). See: https://creativecommons.org/licenses/by-ncnd/4.0/.

\section{References}

1. Engelhardt T, Webster NR. Pulmonary aspiration of gastric contents in anaesthesia. Br J Anaesth 1999;83:453-60.

2. Abdulla S. Pulmonary aspiration in perioperative medicine. Acta Anaesthesiol Belg 2013;64:1-13.

3. Tan Z, Lee SY. Pulmonary aspiration under GA: a 13year audit in a tertiary pediatric unit. Paediatr Anaesth 2016;26:547-52.

4. Eisler L, Huang G, Lee KM, et al. Identification of perioperative pulmonary aspiration in children using quality assurance and hospital administrative billing data. Paediatr Anaesth 2018;28:218-25.

5. Beck CE, Rudolph D, Mahn C, et al. Impact of clear fluid fasting on pulmonary aspiration in children undergoing general anesthesia: Results of the German prospective multicenter observational (NiKs) study. Paediatr Anaesth 2020;30:892-9.

6. Pfaff KE, Tumin D, Miller R, et al. Perioperative aspiration events in children: A report from the Wake Up Safe Collaborative. Paediatr Anaesth 2020;30:660-6.

7. Punjasawadwong $Y$, Srisawasdi S, Werawatganon T, et al. The Thai Anesthesia Incidents Study (THAI study) of ambulatory anesthesia: II. Anesthetic profiles and adverse events. J Med Assoc Thai 2008;91:188-95.

8. Landreau B, Odin I, Nathan N. Pulmonary aspiration: epidemiology and risk factors. Ann Fr Anesth Reanim 2009;28:206-10.

9. El-Orbany M, Connolly LA. Rapid sequence induction and intubation: current controversy. Anesth Analg 2010;110:1318-25.

10. Zdravkovic M, Berger-Estilita J, Sorbello M, et al. An international survey about rapid sequence intubation of 10,003 anaesthetists and 16 airway experts. Anaesthesia 2020;75:313-22.

11. Warner MA, Warner ME, Weber JG. Clinical significance of pulmonary aspiration during the perioperative period. Anesthesiology 1993;78:56-62.

12. Walker RW. Pulmonary aspiration in pediatric anesthetic practice in the UK: a prospective survey of specialist pediatric centers over a one-year period. Paediatr Anaesth 2013;23:702-11.

13. Neelakanta G, Chikyarappa A. A review of patients with pulmonary aspiration of gastric contents during anesthesia reported to the Departmental Quality Assurance Committee. J Clin Anesth 2006;18:102-7.

14. Sakai T, Planinsic RM, Quinlan JJ, et al. The incidence 
and outcome of perioperative pulmonary aspiration in a university hospital: a 4-year retrospective analysis. Anesth Analg 2006;103:941-7.

15. Kelly CJ, Walker RW. Perioperative pulmonary aspiration is infrequent and low risk in pediatric anesthetic practice. Paediatr Anaesth 2015;25:36-43.

16. Bouvet L, Cercueil E, Barnoud S, et al. Relationship between the regurgitated and the aspirated volume of water. A manikin study. Anaesthesiol Intensive Ther 2019;51:121-5.

17. Warner MA, Warner ME, Warner DO, et al. Perioperative pulmonary aspiration in infants and children. Anesthesiology 1999;90:66-71.

18. Olsson GL, Hallen B, Hambraeus-Jonzon K. Aspiration during anaesthesia: a computer-aided study of 185,358 anaesthetics. Acta Anaesthesiol Scand 1986;30:84-92.

19. Forbes JM, Cooper ME. Mechanisms of diabetic complications. Physiol Rev 2013;93:137-88.

20. Bouvet L, Desgranges FP, Aubergy C, et al. Prevalence and factors predictive of full stomach in elective and emergency surgical patients: a prospective cohort study. $\mathrm{Br}$ J Anaesth 2017;118:372-9.

21. Zhou L, Yang Y, Yang L, et al. Point-of-care ultrasound defines gastric content in elective surgical patients with type 2 diabetes mellitus: a prospective cohort study. BMC Anesthesiol 2019;19:179.

22. Ng A, Smith G. Gastroesophageal reflux and aspiration

Cite this article as: Sun J, Wei G, Hu L, Liu C, Ding Z. Perioperative pulmonary aspiration and regurgitation without aspiration in adults: a retrospective observational study of 166,491 anesthesia records. Ann Palliat Med 2021;10(4):4037-4046. doi: 10.21037/apm-20-2382 of gastric contents in anesthetic practice. Anesth Analg 2001;93:494-513.

23. Manning BJ, Winter DC, McGreal G, et al. Nasogastric intubation causes gastroesophageal reflux in patients undergoing elective laparotomy. Surgery 2001;130:788-91.

24. Sun J, Li XH, Zuo YX. Comparison of Incidence of hypoxia during modified rapid sequence induction and an alternative technique: a prospective randomized controlled trial. Int J Clin Exp Med 2015;8:16231-7.

25. Kemper ME, Buehler PK, Schmitz A, et al. Classical versus controlled rapid sequence induction and intubation in children with bleeding tonsils (a retrospective audit). Acta Anaesthesiol Scand 2020;64:41-7.

26. Janda M, Scheeren TW, Nöldge-Schomburg GF. Management of pulmonary aspiration. Best Pract Res Clin Anaesthesiol 2006;20:409-27.

27. Beck-Schimmer B, Bonvini JM. Bronchoaspiration: incidence, consequences and management. Eur J Anaesthesiol 2011;28:78-84.

28. Kluger MT, Short TG. Aspiration during anaesthesia: a review of 133 cases from the Australian Anaesthetic Incident Monitoring Study (AIMS). Anaesthesia 1999;54:19-26.

29. Kluger MT, Visvanathan T, Myburgh JA, et al. Crisis management during anaesthesia: regurgitation, vomiting, and aspiration. Qual Saf Health Care 2005;14:e4. 
Supplementary

Table S1 Details of classic and modified rapid sequence induction

\begin{tabular}{|c|c|c|}
\hline Concerns & $\begin{array}{l}\text { Classic rapid sequence } \\
\text { induction }\end{array}$ & Modified rapid sequence induction \\
\hline Patient position & Supine & $\begin{array}{l}\text { Reverse Trendelenburg or semi-upright position with operation table inclined at an } \\
\text { angle of } 30 \text { to } 45 \text { degrees }\end{array}$ \\
\hline Pre-oxygenation & \multicolumn{2}{|c|}{ Pre-oxygenated by facemask with oxygen flow rate over $10 \mathrm{~L} / \mathrm{min}$ for 3 minutes or more } \\
\hline Drug use & \multicolumn{2}{|c|}{$\begin{array}{l}\text { Induction agents are injected with a smooth infusion in following sequence: propofol at a dose of } \\
1.5-2.5 \mathrm{mg} / \mathrm{kg} \text { or etomidate } 0.25-0.3 \mathrm{mg} / \mathrm{kg} \text {, rocuronium at a dose of } 0.6-1.0 \mathrm{mg} / \mathrm{kg} \text {, fentanyl at a dose of } \\
4.0-6.0 \mu \mathrm{g} / \mathrm{kg} \text { or remifentanil at a dose of } 2.0-4.0 \mu \mathrm{g} / \mathrm{kg} \text {. The vasoactive drugs are delivered as needed. }\end{array}$} \\
\hline $\begin{array}{l}\text { Cricoid pressure } \\
\text { and apneic } \\
\text { oxygenation }\end{array}$ & $\begin{array}{l}\text { Cricoid pressure is applied } \\
\text { as patient lost response. } \\
\text { Apneic oxygenation is not } \\
\text { used }\end{array}$ & $\begin{array}{l}\text { Jaw thrust is performed as patient lost response, and oxygen is continuously } \\
\text { delivered at a flow rate about } 2.0-3.0 \mathrm{~L} / \mathrm{min} \text { with face mask gently put on the patient } \\
\text { face. Cricoid pressure is not applied until lung ventilation is needed }\end{array}$ \\
\hline Tracheal intubation & \multicolumn{2}{|c|}{$\begin{array}{l}\text { Tracheal intubation is finished with video-assisted technique about } 60-90 \text { seconds after rocuronium injected. Skillec } \\
\text { resident or anesthesia nurse is needed for intubation procedure }\end{array}$} \\
\hline
\end{tabular}


Table S2 Demographic data of study population

\begin{tabular}{|c|c|}
\hline Factors & Number of patients (\%) \\
\hline \multicolumn{2}{|l|}{ Gender } \\
\hline Male & $92300(55.4 \%)$ \\
\hline Female & $74191(44.6 \%)$ \\
\hline \multicolumn{2}{|l|}{ Age } \\
\hline $18-64$ years & $119092(71.5 \%)$ \\
\hline$\geq 65$ years & $47399(28.5 \%)$ \\
\hline \multicolumn{2}{|l|}{ ASA grade } \\
\hline I & $25012(15.0 \%)$ \\
\hline II & 109793 (65.9 \%) \\
\hline III & $26679(16.0 \%)$ \\
\hline IV & $4470(2.7 \%)$ \\
\hline V & $537(0.3 \%)$ \\
\hline \multicolumn{2}{|l|}{ Type of admission } \\
\hline Emergency & $10661(6.4 \%)$ \\
\hline Elective & 155830 (93.6 \%) \\
\hline \multicolumn{2}{|l|}{ Type of anesthesia } \\
\hline $\mathrm{GA}(\mathrm{ETT})$ & 156534 (94.0 \%) \\
\hline GA (LMA) & $8494(5.1 \%)$ \\
\hline Regional anesthesia \& MAC & $1463(0.9 \%)$ \\
\hline Total & 166491 \\
\hline
\end{tabular}

ASA: American Society of Anesthesiologist; GA: general anesthesia; ETT: endotracheal tube; LMA: laryngeal mask airway; MAC: monitored anesthesia care.
Table S3 Exclusion reasons for the patients that were ruled out the diagnosis of perioperative regurgitation events

\begin{tabular}{lc}
\hline Reasons for exclusion & Excluded cases (n) \\
\hline $\begin{array}{l}\text { Recording "regurgitation/aspiration not } \\
\text { occurred" }\end{array}$ & 120 \\
$\begin{array}{l}\text { Regurgitation/aspiration occurred before } \\
\text { admitting }\end{array}$ & 83 \\
Gastric tube suctioning for surgical demands & 42 \\
Postoperative nausea and vomiting & 13 \\
Respiratory secretions & 1 \\
Biliary bronchial fistula & 1 \\
Total & 260 \\
\hline
\end{tabular}


Table S4 Detailed information of patients with pulmonary aspiration and regurgitation without aspiration

\begin{tabular}{|c|c|c|c|c|c|c|c|c|c|c|c|c|}
\hline No. & Gender & Age (yr) & ASA grade & Risk factors & Diagnosis & RSI & Airway management & Time of occurrence & PA/R & Treatment & Postoperative mechanical ventilation & Outcome \\
\hline 1 & $M$ & 89 & 4 & E, acute abdomen, sepsis & Postoperative biliary fistula & $\mathrm{N}$ & ETT & Induction & PA & $\mathrm{H}$ & Scheduled & ICU, death \\
\hline 2 & M & 46 & 4 & E, RM & Tracheal stenosis & $\mathrm{Y}$ & ETT & Induction & PA & BS & Scheduled & $\mathrm{ICU}$, discharge \\
\hline 3 & M & 67 & 5 & $E$, acute abdomen & Gastrointestinal perforation & $\mathrm{Y}$ & ETT & Induction & $\mathrm{PA}(\mathrm{s})$ & None & Scheduled & NW, death \\
\hline 4 & $\mathrm{~F}$ & 47 & 3 & $E$, acute abdomen & Gastrointestinal perforation & Y & ETT & Induction & PA & $B L, B S, H$ & Scheduled & $\mathrm{ICU}$, discharge \\
\hline 5 & M & 49 & 2 & None & Hepatoma & $\mathrm{N}$ & ETT & Induction & $\mathrm{PA}(\mathrm{s})$ & $\mathrm{BL}, \mathrm{PEEP}, \mathrm{AB}, \mathrm{H}$ & None & NW, discharge \\
\hline 6 & M & 54 & 3 & None & Abdominal space-occupying lesion & $\mathrm{N}$ & ЕT & Induction & PA & PEEP, $\mathrm{H}$ & PA related & $\mathrm{ICU}^{\dagger}$, discharge \\
\hline 7 & M & 75 & 4 & Previous esophageal surgery & Postoperative thoracic infection & $\mathrm{Y}$ & ETT & Induction & PA & $B S, H$ & Scheduled & $\mathrm{ICU}$, discharge \\
\hline 8 & M & 28 & 5 & $\mathrm{E}$ & Pancreatic transection & $\mathrm{Y}$ & ЕT & Induction & PA & None & Scheduled & $\mathrm{ICU}$, discharge \\
\hline 9 & M & 49 & 4 & E, RM, drowsiness & Splenic rupture & $\mathrm{Y}$ & ETT & Induction & PA & None & Scheduled & $\mathrm{ICU}$, discharge \\
\hline 10 & M & 48 & 2 & BMI 29.4 & Renal calculus & $\mathrm{N}$ & ETT & Induction & PA & $B L, H$ & None & NW, discharge \\
\hline 11 & M & 78 & 4 & $E, R M$, incarcerated inguinal hernia & Incarcerated inguinal hernia & $\mathrm{Y}$ & ЕTT & Induction & PA & BL & PA related & $\mathrm{ICU}^{\dagger}$, death $^{\dagger}$ \\
\hline 12 & M & 45 & 2 & E, RM & Hand trauma & $\mathrm{Y}$ & ЕT & Induction & $\mathrm{PA}(\mathrm{s})$ & $\mathrm{H}$ & None & NW, discharge \\
\hline 13 & M & 76 & 3 & $E, R M$, intestinal obstruction, renal failure & Intestinal obstruction & $\mathrm{Y}$ & ETT & Induction & PA & $\mathrm{BL}$ & None & NW, discharge \\
\hline 14 & M & 45 & 2 & BMI 29.4 & Gallstone & $\mathrm{N}$ & ETT & Induction & PA & BL, BS, PEEP, H & None & NW, discharge \\
\hline 15 & M & 65 & 3 & Previous esophageal surgery & Inguinal Hernia & $\mathrm{Y}$ & ETT & Induction & PA & $B L, B S, A B, H$ & PA related & $\mathrm{ICU}^{\dagger}$, discharge \\
\hline 16 & M & 69 & 5 & $\mathrm{E}$ & Cerebral hemorrhage & $\mathrm{Y}$ & ЕT & Induction & PA & $\mathrm{H}$ & Scheduled & NW, discharge \\
\hline 17 & M & 50 & 2 & BMI 27.8 & Varicocele & $\mathrm{N}$ & ETT & Induction & PA & BL, BS, PEEP, AP; H & None & NW, discharge \\
\hline 18 & $\mathrm{~F}$ & 73 & 3 & $E$, previous esophageal surgery & Tracheal stenosis & $\mathrm{N}$ & LMA & Induction & PA & BS, $\mathrm{H}$ & PA related & $\mathrm{ICU}^{\dagger}$, discharge \\
\hline 19 & M & 76 & 3 & E, intestinal obstruction & Intestinal obstruction & Y & ЕTT & Prior to induction & PA & $\mathrm{H}$ & None & $N W$, death $^{\dagger}$ \\
\hline 20 & M & 74 & 3 & $E$, intestinal obstruction & Intestinal obstruction & $\mathrm{Y}$ & ETT & Induction & PA & $B L, B S, A P ; H$ & PA related & $\mathrm{ICU}^{\dagger}$, death ${ }^{\dagger}$ \\
\hline 21 & M & 50 & 2 & $E, R M$, incarcerated inguinal hernia & Incarcerated inguinal hernia & $\mathrm{Y}$ & ETT & Prior to induction & $\mathrm{R}$ & H & None & NW, discharge \\
\hline 22 & M & 27 & 1 & E, acute abdomen, BMI 31.2 & Acute appendicitis & $\mathrm{N}$ & ETT & Induction & $\mathrm{R}$ & None & None & NW, discharge \\
\hline 23 & M & 28 & 5 & $\mathrm{E}, \mathrm{RM}$ & Haematemesis & $\mathrm{N}$ & ЕT & Prior to induction & $\mathrm{R}$ & BS & Scheduled & ICU, death \\
\hline 24 & $\mathrm{~F}$ & 64 & 4 & $E$, coma & Cerebral hemorrhage & $\mathrm{Y}$ & ETT & Emergence & $\mathrm{R}$ & None & Scheduled & NW, discharge \\
\hline 25 & M & 20 & 3 & $\mathrm{E}, \mathrm{RM}$ & Pseudoaneurysm & $\mathrm{Y}$ & ETT & Prior to induction & $\mathrm{R}$ & None & None & NW, discharge \\
\hline 26 & M & 53 & 4 & E, RM, coma & Cerebral hemorrhage & $\mathrm{Y}$ & ЕT & Prior to induction & $\mathrm{R}$ & $\mathrm{H}$ & Scheduled & $\mathrm{ICU}$, discharge \\
\hline 27 & M & 51 & 5 & E, RM & Aortic dissection & $\mathrm{N}$ & ЕT & Induction & $\mathrm{R}$ & None & Scheduled & NW, death \\
\hline 28 & M & 74 & 4 & $\mathrm{E}$, coma & Cerebral trauma & Y & ЕT & Prior to induction & $\mathrm{R}$ & None & Scheduled & $\mathrm{ICU}$, death \\
\hline 29 & M & 80 & 2 & $\mathrm{E}, \mathrm{RM}$, acute abdomen & Acute appendicitis & $\mathrm{Y}$ & ETT & Prior to induction & $\mathrm{R}$ & None & None & NW, discharge \\
\hline 30 & M & 60 & 2 & $\mathrm{RM}$ & Hepatic space-occupying lesion & $\mathrm{Y}$ & ETT & Prior to induction & $\mathrm{R}$ & None & None & NW, discharge \\
\hline 31 & M & 66 & 3 & $\mathrm{E}, \mathrm{RM}$, Intestinal obstruction & Intestinal volvulus & $\mathrm{Y}$ & ЕT & Prior to induction & $\mathrm{R}$ & None & None & NW, discharge \\
\hline 32 & M & 53 & 2 & E, RM, acute abdomen & Gastrointestinal perforation & $\mathrm{N}$ & ЕT & Induction & $\mathrm{R}$ & None & None & NW, discharge \\
\hline 33 & M & 79 & 4 & E, RM, acute abdomen & Gastrointestinal perforation & $\mathrm{Y}$ & ETT & Induction & $\mathrm{R}$ & None & Scheduled & NW, discharge \\
\hline 34 & M & 51 & 1 & None & Colon cancer & $\mathrm{N}$ & ETT & Maintenance & $\mathrm{R}$ & None & None & NW, discharge \\
\hline 35 & M & 69 & 3 & None & Prostate cancer & $\mathrm{N}$ & ETT & Maintenance & $\mathrm{R}$ & None & None & NW, discharge \\
\hline 36 & M & 65 & 3 & $E, R M$, sepsis & Acute appendicitis & $\mathrm{Y}$ & ЕT & Induction & $\mathrm{R}$ & None & None & NW, discharge \\
\hline 37 & M & 66 & 4 & $E, R M$, acute abdomen, sepsis, drowsiness & Gastrointestinal perforation & $\mathrm{N}$ & ЕT & Induction & $\mathrm{R}$ & $\mathrm{H}$ & Scheduled & $\mathrm{ICU}$, discharge \\
\hline 38 & M & 66 & 2 & E, RM, Intestinal obstruction & Intestinal obstruction & $\mathrm{Y}$ & ETT & Maintenance & $\mathrm{R}$ & None & None & NW, discharge \\
\hline 39 & M & 84 & 3 & $\mathrm{E}, \mathrm{RM}, \mathrm{coma}$ & Extensive burns & $\mathrm{N}$ & ETT & Maintenance & $\mathrm{R}$ & None & Scheduled & NW, discharge \\
\hline 40 & M & 31 & 4 & $E$, RM, drowsiness & Cerebral trauma & $\mathrm{Y}$ & ETT & Prior to induction & $\mathrm{R}$ & $B S, H$ & Scheduled & ICU, discharge \\
\hline
\end{tabular}

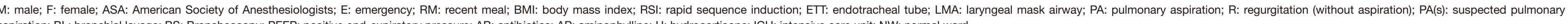


Table S5 Surgical specialized incidence of pulmonary aspiration and regurgitation without aspiration

\begin{tabular}{|c|c|c|}
\hline Surgical departments & PA & $\mathrm{R}$ \\
\hline General $^{\dagger}$ & 13/56058 (1:4312) & $12 / 56058(1: 4672)$ \\
\hline Urology & $2 / 22963$ (1:11482) & 1/22963 (1:22963) \\
\hline Neurosurgery & $1 / 11590(1: 11590)$ & 4/11590 (1:2898) \\
\hline Orthopedic & 1/29227 (1:29227) & 0/29227 (0:29227) \\
\hline Vascular & 0/2256 (0:2256) & $1 / 2256(1: 2256)$ \\
\hline Cardiac & 0/6828 (0:6828) & $1 / 6828(1: 6828)$ \\
\hline Ear nose throat & $0 / 10400(0: 10400)$ & $1 / 10400(1: 10400)$ \\
\hline Ophthalmology & 0/2102 (0:2102) & 0/2102 (0:2102) \\
\hline Others & 0/1583 (0:1583) & 0/1583 (0:1583) \\
\hline Total & 20/166491 (1:10184) & $20 / 166491(1: 8856)$ \\
\hline
\end{tabular}

PA: pulmonary aspiration; R: regurgitation (without aspiration). ${ }^{\dagger}$ Department of general surgery includes gastrointestinal, hepatobiliary, colorectum, thyroid and breast surgery.

Table S6 Higher ASA grades were found in patients with pulmonary aspiration and regurgitation without aspiration

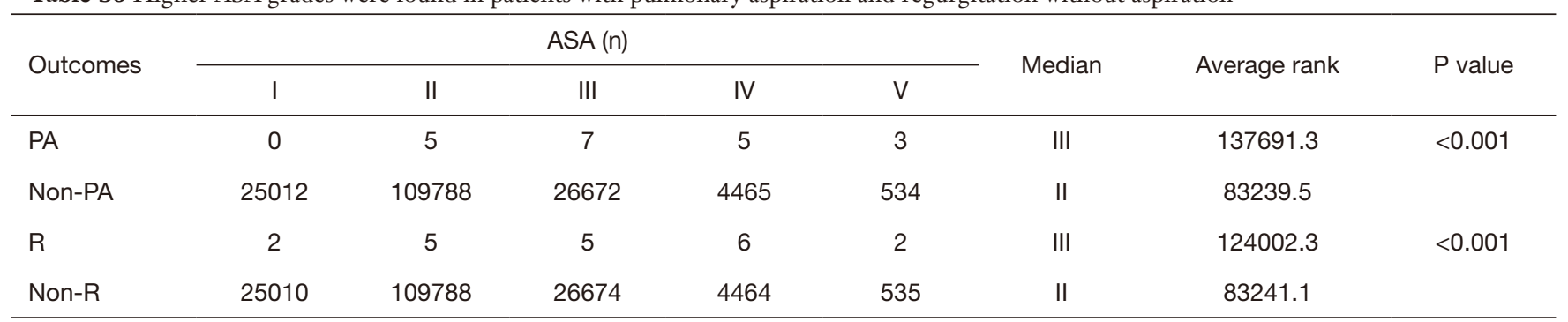

PA: pulmonary aspiration; R: regurgitation (without aspiration). ASA grades were compared by Mann-Whitney U test.

Table S7 Sample size estimation for future study

\begin{tabular}{lcccccc}
\hline$P_{m}$ & $P_{c}$ & $\alpha$ & $1-\beta$ & Allocation ratio & $S_{m}$ & $S_{c}$ \\
\hline $1 / 1055$ & $12 / 4469$ & 0.05 & 0.8 & $1: 2$ & 7484 & 14968 \\
\hline
\end{tabular}

$\mathrm{P}_{\mathrm{m}}$ : proportion of modified RSI group; $\mathrm{P}_{\mathrm{c}}$ : proportion of classic RSI group; $\mathrm{S}_{\mathrm{m}}$ : sample size of modified RSI group; $\mathrm{S}_{\mathrm{c}}$ : sample size of classic RSI group. $P_{m}$ and $P_{c}$ were based on our current incidences of regurgitation events in emergency patients who received modified or classic RSI protocol. Since 'proportion' must be set between 0 and 1, $P_{m}$ was set 1/1055 instead of 0/1055. With the extensive application of modified RSI protocol, the allocation ratio was set 1:2 (>1055/4469). 
Table S8 Details of the three patients who died of perioperative pulmonary aspiration

No. Details of pulmonary aspiration and postoperative course

11 Pulmonary aspiration occurred during induction and was confirmed by blind suctioning. Chest radiograph reported a newly detected multiple patchy of both lungs later in the day of surgery. The patient was diagnosed with ARDS with pneumonia of multidrug-resistant acinetobacter baumannii. He died of severe hypoxemia and cardiac arrest 12 days postoperatively.

19 Pulmonary aspiration occurred before induction and was confirmed by blind suctioning. The patient was extubated after surgery and returned normal ward with a pulse oxygen saturation of $93 \%$ breathing room air. But he developed progressively worse hypoxemia approximately 10 hours after surgery. He was then transferred to ICU for bronchial lavage and anti-infection but finally died of multiple organ failure 12 days after surgery.

20 Pulmonary aspiration occurred during induction and was confirmed by bronchoscopy. Chest radiograph reported a newly detected multiple patchy of both lungs the day after surgery. The patient received bronchial lavage in ICU with limited improvement on hypoxemia. He experienced supraventricular tachycardia and cardiac arrest the day after surgery and his family members abandoned invasive methods of rescue.

Patient number is in accordance with that in Table S3. 